\title{
Crystal Structure and Magnetic Properties of Pyrrhotite-Type Compounds $\mathrm{Fe}_{7-y} \mathrm{~V}_{y} \mathrm{~S}_{8}$
}

\author{
N.V. Selezneva ${ }^{a, *}$, P.N.G. Ibrahim ${ }^{a}$, N.M. Toporova ${ }^{a}$, E.M. Sherokalova $^{a}$ \\ AND N.V. BARANOV ${ }^{a, b}$ \\ ${ }^{a}$ Institute of Natural Sciences and Mathematics, Ural Federal University, 620083, Ekaterinburg, Russia \\ ${ }^{b}$ M.N. Miheev Institute of Metal Physics, Ural Branch of RAS, 620990, Ekaterinburg, Russia
}

The pyrrhotite-type compounds $\mathrm{Fe}_{7-y} \mathrm{~V}_{y} \mathrm{~S}_{8}$ have been synthesized and studied by means of the X-ray diffraction and magnetization measurements in order to reveal how the substitution of $\mathrm{V}$ for $\mathrm{Fe}$ affects the crystal structure, phase transition and magnetic properties. The growth of the $\mathrm{V}$ content in $\mathrm{Fe}_{7-y} \mathrm{~V}_{y} \mathrm{~S}_{8}$ results in changes of the crystal structure and leads to a sharp decrease in the resultant magnetization, non-monotonous change of the coercive field and reduction of the magnetic ordering temperature. Unlike ferrimagnetic ordering in pyrrhotite $\mathrm{Fe}_{7} \mathrm{~S}_{8}$ the compound $\mathrm{V}_{7} \mathrm{~S}_{8}$ is observed to exhibit a Pauli-paramagnetic behaviour.

DOI: 10.12693/APhysPolA.133.450

PACS/topics: $61.66 . \mathrm{Fn}, 75.30 . \mathrm{Kz}, 75.50 . \mathrm{Gg}$

\section{Introduction}

Pyrrhotite $\left(\mathrm{Fe}_{7} \mathrm{~S}_{8}\right)$ consists of completely filled layers of chalcogen sandwiched between Fe layers one of which is full, while another one contains vacancies [1]. The $\mathrm{Fe}$ magnetic moments $\left(\mu_{\mathrm{Fe}} \sim 3.2 \mu_{\mathrm{B}}\right)$ are arranged parallel to each other inside each layer, but are coupled antiparellel to each other between successive layers [2]. The presence of vacancies in every second Fe layer leads to incomplete compensation of magnetic moments and to a ferrimagnetic order which enters below the Neel temperature of about $590 \mathrm{~K}[1,2]$. The magnetic properties of $\mathrm{Fe}_{7} \mathrm{X}_{8}$ are strongly influenced by substitutions in both the $\mathrm{Fe}[3,4]$ and chalcogen sublattices [5]. The replacement of iron in $\mathrm{Fe}_{7-y} \mathrm{M}_{y} \mathrm{X}_{8}(\mathrm{X}=\mathrm{S}, \mathrm{Se})$ by other $3 \mathrm{~d}$ metal $(\mathrm{M}=\mathrm{Ti}, \mathrm{Co})$ atoms in cation layers is found to be non-random and dramatically affects the magnetic behavior [4]. The cation partitioning between layers in pyrrhotite-type compounds is observed to depend on the kind of substituting $\mathrm{M}$ atoms assumingly owing to the difference in the spatial extension of 3d orbitals [4]. In order to confirm this suggestion further the present work aims to study how the substitution of $\mathrm{V}$ for Fe affects the properties of the $\mathrm{Fe}_{7-y} \mathrm{~V}_{y} \mathrm{~S}_{8}$ compounds.

\section{Experimental}

Polycrystalline samples $\mathrm{Fe}_{7-y} \mathrm{~V}_{y} \mathrm{~S}_{8}(y=0-7)$ were obtained by solid-state reactions in evacuated quartz tubes. The starting materials were small vanadium pieces $(99.9 \%$ purity), sulfur $(99.99 \%)$ and powder of iron (99.98\%). The method of synthesis of $\mathrm{Fe}_{7-y} \mathrm{M}_{y} \mathrm{~S}_{8}$ samples has already been described in detail [4]. In order to examine the quality of the samples and the changes in the crystal structure upon substitution a powder X-ray

*corresponding author; e-mail: hope_s@mail.ru diffractometer Bruker D8 ADVANCE with $\mathrm{Cu}$ K $\alpha$ radiation was used. The measurements of the magnetic susceptibility and magnetization were performed by means of a Quantum Design SQUID MPMS magnetometer and Vibrating sample magnetometer Lake Shore VSM 7407.

\section{Results}

According to X-ray diffraction all the synthesized samples $\mathrm{Fe}_{7-y} \mathrm{~V}_{y} \mathrm{~S}_{8}(0 \leqslant y \leqslant 7)$ have layered superstructures of the NiAs-type, while in the case of Ti for Fe substitution, the single phase $\mathrm{Fe}_{7-y} \mathrm{Ti}_{y} \mathrm{~S}_{8}$ samples were obtained only at $y \leqslant 4[3]$. The changes in the crystal structure in $\mathrm{Fe}_{7-y} \mathrm{~V}_{y} \mathrm{~S}_{8}$ with substitution can be characterized by the lattice parameters $a_{0}$ and $c_{0}$ of the NiAs fundamental unit cell. The $\mathrm{V}$ for Fe substitution is found to result in the growth of the $c_{0}$ value from 5.668(6) $\AA$ in $\mathrm{Fe}_{7} \mathrm{~S}_{8}$ $(y=0)$ up to $5.789(2) \AA$ in $\mathrm{V}_{7} \mathrm{~S}_{8}(y=7)$, however, the average intra-layer distance characterized by lattice parameter $a_{0}$ slightly decreases with increasing $\mathrm{V}$ content from 3.436(1) $\AA$ for $y=0$ down to 3.376(3) $\AA$ for $y=7$.

The unit cell volume remains almost unchanged upon substitution in the $\mathrm{Fe}_{7-y} \mathrm{~V}_{y} \mathrm{~S}_{8}$ system.

The replacement of Fe by $\mathrm{V}$ atoms is observed to dramatically affect the magnetization behaviour in magnetic fields (shown in Fig. 1). As in the case of the $\mathrm{Ti}$ for $\mathrm{Fe}$ substitution $[3,4]$, the replacement of $\mathrm{Fe}$ by $\mathrm{V}$ up to $y=1$ significantly reduces the magnetization, while further growth of the $\mathrm{V}$ content results in a non-monotonous change of the magnetization. Fig. 2a shows the concentration dependence of the magnetization $M_{50}$ measured in a field of $50 \mathrm{kOe}$ at temperature $2 \mathrm{~K}$ on the $\mathrm{Fe}_{7-y} \mathrm{~V}_{y} \mathrm{~S}_{8}$ samples. The non-monotonous behaviour of the magnetization was also observed in the $\mathrm{Fe}_{7-y} \mathrm{Ti}_{y} \mathrm{~S}_{8}$ system, which was explained by the ferrimagnetic ordering of the Fe magnetic moments and by non-random substitutions in the cation layers $[3,4]$ since the statistical distribution of substituting atoms should result in the monotonous reduction of the resultant magnetization. 


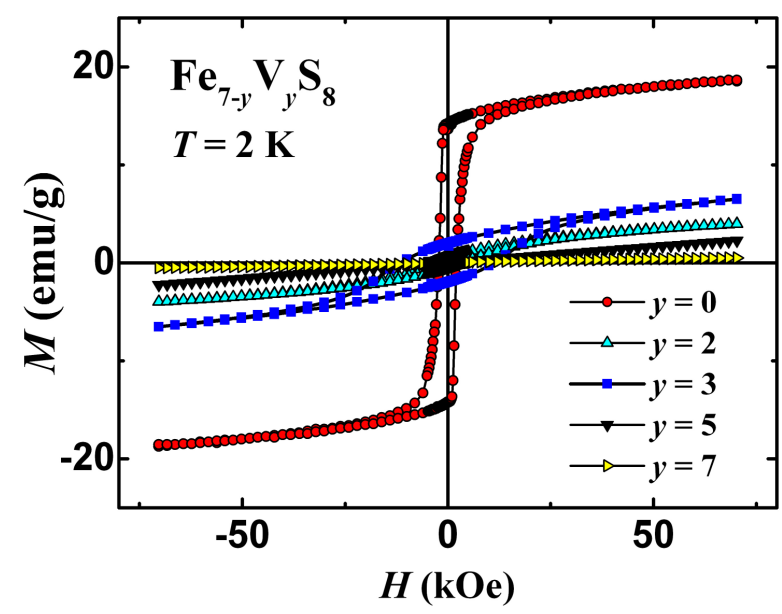

Fig. 1. Field dependences of the magnetization measured at $T=2 \mathrm{~K}$ on the $\mathrm{Fe}_{7-y} \mathrm{~V}_{y} \mathrm{~S}_{8}$ samples.

For $\mathrm{Fe}_{7-y} \mathrm{Ti}_{y} \mathrm{~S}_{8}$, the non-random $\mathrm{Ti}$ for Fe substitution in alternating cationic layers was derived from neutron diffraction data [4]. The presence of a minimum of the resultant magnetization at $y \sim 1.5$ in $\mathrm{Fe}_{7-y} \mathrm{~V}_{y} \mathrm{~S}_{8}$ may be associated with the compensation of the sublattice magnetizations, as in $\mathrm{Fe}_{7-y} \mathrm{Ti}_{y} \mathrm{~S}_{8}[3,4]$. However, unlike the $\mathrm{Fe}_{7-y} \mathrm{Ti}_{y} \mathrm{~S}_{8}$ samples with $y>1.5$ exhibiting almost in twice increased magnetization in high magnetic fields in comparison with the non-substituted $\mathrm{Fe}_{7} \mathrm{~S}_{8}$ compound, all the $\mathrm{Fe}_{7-y} \mathrm{~V}_{y} \mathrm{~S}_{8}$ compounds demonstrate substantially lower values of the resultant magnetization. There are several origins which may lead to reduced values of the magnetization in the V-substituted compounds: i) the less pronounced partitioning of $\mathrm{Fe}$ and $\mathrm{V}$ between adjacent layers; ii) the more random distribution of vacancies in all cationic layers and iii) presence of a non-zero magnetic moment on $\mathrm{V}$ ions unlike Ti. The neutron diffraction measurements are needed in order to reveal the main factors determining the magnetization behavior with increasing $\mathrm{V}$ content in $\mathrm{Fe}_{7-y} \mathrm{~V}_{y} \mathrm{~S}_{8}$.

As follows from Fig. 2b, the substitution of $\mathrm{V}$ for $\mathrm{Fe}$ in $\mathrm{Fe}_{7-y} \mathrm{~V}_{y} \mathrm{~S}_{8}$ leads to the non-monotonous variations of the coercive field $\left(H_{c}\right)$, as in $\mathrm{Fe}_{7-y} \mathrm{Ti}_{y} \mathrm{~S}_{8}$. The growth of $H_{c}$ around $y=1-1.5$ in both the $\mathrm{V}$ - and Ti-containing compounds apparently originates in the reduced values of magnetization. Such a behavior is analogous to that observed in vicinity of the compensation temperature in ferrimagnetic materials. When the $\mathrm{V}$ content increases up to $y=3$, the hysteresis loop becomes broader and $H_{c}$ reaches a value about of $11.5 \mathrm{kOe}$; further substitution of $\mathrm{V}$ for $\mathrm{Fe}$ reduces the hysteresis and leads to the paramagnetic behavior of $\mathrm{Fe}_{7-y} \mathrm{~V}_{y} \mathrm{~S}_{8}$ at $y>6$.

The magnetic state of the $\mathrm{V}$-substituted compounds $\mathrm{Fe}_{7-y} \mathrm{~V}_{y} \mathrm{~S}_{8}$ with $y \sim 5$ seems to be non-homogeneous. The temperature dependence of the magnetic susceptibility of $\mathrm{Fe}_{2} \mathrm{~V}_{5} \mathrm{~S}_{8}$ (Fig. 3a) shows a remarkable hysteresis at low temperatures when the measurements were made on a sample cooled in zero field (ZFC) or on a sample cooled in an applied field (FC).

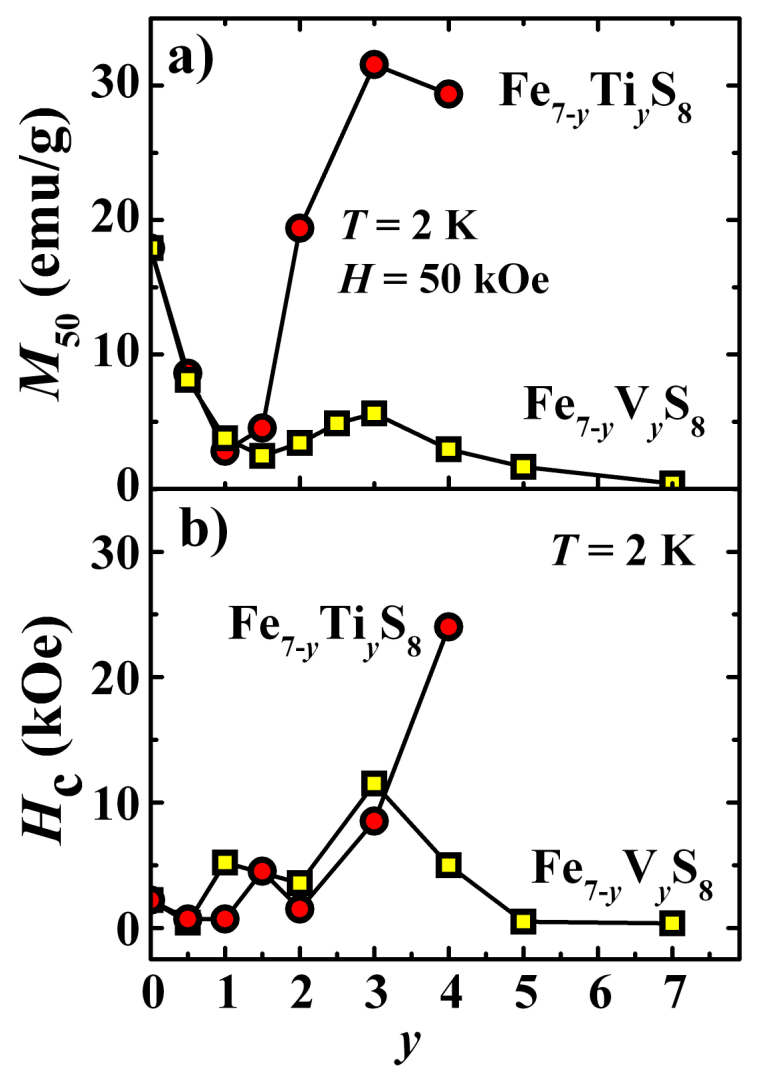

Fig. 2. Magnetization (a) in a field of $50 \mathrm{kOe}$ and coercive field (b) for $\mathrm{Fe}_{7-y} \mathrm{M}_{y} \mathrm{~S}_{8}(\mathrm{M}=\mathrm{V}, \mathrm{Ti})$ at $T=2 \mathrm{~K}$ as functions of the substituting atom concentrations. The data for $\mathrm{Fe}_{7-y} \mathrm{Ti}_{y} \mathrm{~S}_{8}$ are taken from Ref. [4].

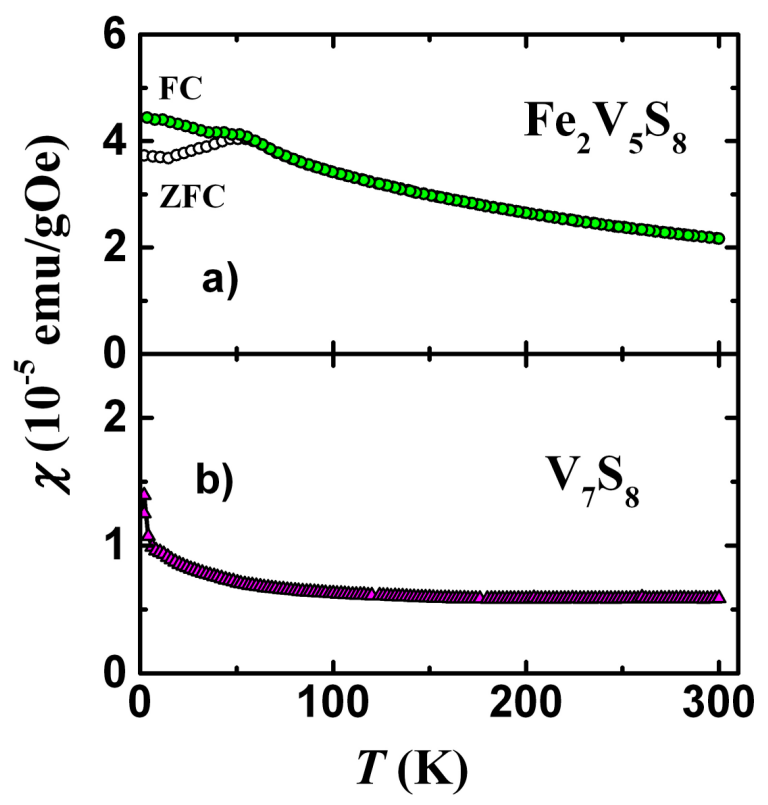

Fig. 3. Temperature dependences of the magnetic susceptibility for $\mathrm{Fe}_{2} \mathrm{~V}_{5} \mathrm{~S}_{8}$ (a) and $\mathrm{V}_{7} \mathrm{~S}_{8}$ (b) measured at $H=1 \mathrm{kOe}$. 
These data together with a reduced magnetization and broad hysteresis loop allow us to characterize the magnetic state of this compound as of cluster glass-type with freezing of the ferrimagnetic clusters below $50 \mathrm{~K}$. As follows from Fig. 3b, the magnetic susceptibility of $\mathrm{V}_{7} \mathrm{~S}_{8}(y=7)$ shows weak temperature dependence above $T \sim 100 \mathrm{~K}$ and a low-temperature upturn of the CurieWeiss (CW) type. Such a paramagnetic behavior of this compound is consistent with previously reported data for the vanadium sulfides with nearly equiatomic compositions [6]. The $\chi(T)$ dependence for $\mathrm{V}_{7} \mathrm{~S}_{8}$ can be fitted to the expression: $\chi(T)=\chi_{0}+C /\left(T-\Theta_{p}\right)$, in which the first term is the temperature-independent Pauli paramagnetic contribution from conduction electrons and the second one is the CW contribution from localized magnetic moments. Form the Curie constant $C$, the effective magnetic moment $\mu_{\text {eff }}$ was estimated to be $0.3 \mu_{\mathrm{B}}$ per $\mathrm{V}$ ion, which is substantially lower than the spin-only values for $\mathrm{V}^{3+}\left(2.83 \mu_{\mathrm{B}}\right)$ and $\mathrm{V}^{4+}\left(1.73 \mu_{\mathrm{B}}\right)$.

Figure 4 shows the variation of the magnetic ordering temperature $\left(T_{N}\right)$ with the $\mathrm{V}$ for Fe substitution in $\mathrm{Fe}_{7-y} \mathrm{~V}_{y} \mathrm{~S}_{8}$ together with the $T_{N}(y)$ dependence for $\mathrm{Fe}_{7-y} \mathrm{Ti}_{y} \mathrm{~S}_{8}$. The Neel temperatures for the V-containing samples at $y<4$ are found to be greater than that for the compounds $\mathrm{Fe}_{7-y} \mathrm{Ti}_{y} \mathrm{~S}_{8}$ with the same concentrations of the substituting elements.

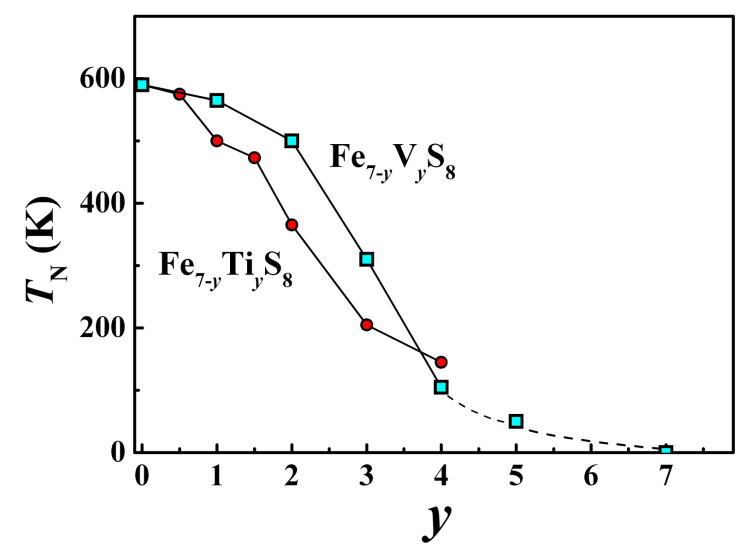

Fig. 4. The concentration dependences of the Neel temperature for $\mathrm{Fe}_{7-y} \mathrm{M}_{y} \mathrm{~S}_{8}(\mathrm{M}=\mathrm{V}, \mathrm{Ti})$. The data for $\mathrm{Fe}_{7-y} \mathrm{Ti}_{y} \mathrm{~S}_{8}$ are taken from Ref. [4].

The enhancement of magnetic ordering temperatures in the $\mathrm{Fe}_{7-y} \mathrm{~V}_{y} \mathrm{~S}_{8}$ system may be indicative of the presence of a local magnetic moment on vanadium atoms in the compounds $\mathrm{Fe}_{7-y} \mathrm{~V}_{y} \mathrm{~S}_{8}$ with $y<4$ in contrast to the titanium atoms in $\mathrm{Fe}_{7-y} \mathrm{Ti}_{y} \mathrm{~S}_{8}$.

\section{Conclusions}

The compounds $\mathrm{Fe}_{7-y} \mathrm{~V}_{y} \mathrm{~S}_{8}$ are found to exhibit the layered crystal structures of the NiAs-type in the whole concentration range. The growth of the $\mathrm{V}$ content increases the average inter-layer distance and reduces the average intra-layer distance, however, the unit cell volume remains almost unchanged upon substitution. The replacement of Fe by $\mathrm{V}$ in $\mathrm{Fe}_{7-y} \mathrm{~V}_{y} \mathrm{~S}_{8}$ having a ferrimagnetic order at $y=0$ leads to a non-monotonous change of the resultant magnetization. A minimum of the magnetization around $y=1.5$ is attributed to the partial compensation of the antiferromagnetically ordered magnetic moments of $3 \mathrm{~d}$ metal ions located in neighbor cationic layers because of non-random substitutions of $\mathrm{V}$ for $\mathrm{Fe}$. The presence of such a minimum indicates that vanadium preferably substitutes iron in layers without vacancies when the $\mathrm{V}$ content increases up to $y=1.5$ as was observed earlier in the case of Ti for Fe substitution [4]. In $\mathrm{Fe}_{7-y} \mathrm{~V}_{y} \mathrm{~S}_{8}$, the coercive field reaches a maximal value $11.5 \mathrm{kOe}$ at $y=1.5$ obviously because of a reduced value of the resultant magnetization at this concentration. Unlike a non-monotonous variation of the resultant magnetization and coercive field the growth of the $\mathrm{V}$ content leads to a monotonous decrease of the magnetic ordering temperature. The $\mathrm{Fe}_{7-y} \mathrm{~V}_{y} \mathrm{~S}_{8}$ compounds are observed to exhibit a cluster-glass behavior within the concentration range $4<y \leqslant 6$. A reduced value of the effective magnetic moment per $\mathrm{V}$ atom in $\mathrm{V}_{7} \mathrm{~S}_{8}\left(\mu_{e f f}=0.3 \mu_{\mathrm{B}}\right)$ indicates that the magnetic state of this compound can not be described in terms of a model with localized d electrons. The $3 \mathrm{~d}$ electrons of $\mathrm{V}$ ions in the $\mathrm{Fe}_{7-y} \mathrm{~V}_{y} \mathrm{~S}_{8}$ system are rather in an intermediate regime between strongly localized and itinerant case.

\section{Acknowledgments}

This work was supported by the RFBR (projects No 16-02-00480 and 16-03-00733), by the Ministry of Education and Science of Russia (project No 3.2916.2017/4.6) and by FASO Russia (theme 01201463328).

\section{References}

[1] H. Wang, I. Salveson, Phase transition 78, 547 (2005).

[2] A.V. Powell, P. Vaqueiro, K.S. Knight, L.C. Chapon, R.D. Sanchez, Phys. Rev. B 70, 014415 (2004).

[3] N.V. Baranov, P. N.G. Ibrahim, N.V. Selezneva, V.A. Kazantsev, A.S. Volegov, D.A. Shishkin, Physica B 449, 229 (2014).

[4] N.V. Baranov, P.N.G. Ibrahim, N.V. Selezneva, A.F. Gubkin, A.S. Volegov, D.A. Shishkin, L. Keller, D. Sheptyakov, E.A. Sherstobitova, J. Phys.: Cond. Matter 27, 286003 (2015).

[5] T. Ericsson, Ö. Amcoff, P. Nordblad, Hyperfine Interactions 90, 515 (1994).

[6] A.B. De Vries, C. Haas, J. Phys. Chem. Sol. 34, 651 (1973). 